\title{
Metodología PARA UN PLAN LOCAL DE SEGURIDAD VIAL - MODOS NO MOTORIZADOS (PLSV-MNM): CASO MEDELLÍN
}

\author{
Juan Manuel Gómez ${ }^{1}$, Sandra Milena Serna ${ }^{2}$ \\ ${ }^{1}$ Especialista de tránsito en Sistemas Inteligentes en Red S.A.S. Correo electrónico: jgomez@sistemasinteligentesenred.com.co \\ ${ }^{2}$ Analista de tránsito en Sistemas Inteligentes en Red S.A.S. \\ Medellín, Colombia
}

Recibido: 27 de septiembre del 2013. Aprobado: 10 de febrero del 2014.

Cómo citar este artículo: J. M. Gómez y S. M. Serna, "Metodología para un plan local de seguridad vial - modos no motorizados (PLSV-MNM): caso Medellín". Ingeniería Solidaria, vol. 10, n. ${ }^{\circ}$ 17, pp. 175-178, en.-dic., 2014. doi: http://dx.doi.org/10.16925/in.v10i17.878

Resumen. De acuerdo con las cifras de accidentalidad registradas entre los años 2006 y 2012 en la ciudad de Medellín, más de la mitad de las personas fallecidas en accidentes de tránsito corresponden a ciclistas y peatones. Por esta razón, las autoridades locales tienen el deber de desarrollar e implementar un plan de seguridad vial, coherente con el Plan Nacional de Seguridad Vial (PNSv 2001-2016), establecido por el Gobierno de Colombia, a través del Ministerio de Transporte, cuyo objetivo es reducir en un 50\% las mortalidades derivadas de los incidentes de tránsito. Con base en esto, se propone una metodología para el diagnóstico, planificación y evaluación de las intervenciones necesarias para la reducción de la mortalidad de los actores más vulnerables en la vía.

Palabras clave: peatones, seguridad vial, modos no motorizados, bicicleta.

\section{METHODOLOGY FOR A LOCAL ROAD SAFETY PLAN - NON- MOTORIZED MODES (PLSV-MNM): CASE OF MEDELLÍN}

Abstract. According to accident figures recorded between 2006 and 2012 in the city of Medellín, more than half of those killed in traffic accidents are bicyclists and pedestrians. That is why local authorities have the duty to develop and implement a road safety plan, coherent with the National Road Safety plan (Plan Nacional de Seguridad Vial, PNsv 2001-2016), established by the Government of Colombia through its Ministry of Transport, with the aim of reducing deaths from traffic incidents by $50 \%$. A methodology is therefore proposed to diagnose, plan and evaluate necessary interventions to reduce the mortality of the most vulnerable actors on the roads.

Keywords: pedestrians, road safety, non-motorized modes, bicycle.

\section{METODOlOgia PARA UM PLANO LOCAL DE SEGURANÇA VIÁRIA - MODOS NÃO MOTORIZADOS (PLSV-MNM): CASO MEDELLÍN}

Resumo. De acordo com as cifras de acidentalidade registradas entre 2006 e 2012 na cidade de Medellín, mais da metade das pessoas falecidas em acidentes de trânsito correspondem a ciclistas e pedestres. Por essa razão, as autoridades locais têm o dever de desenvolver e implementar um plano de segurança viária, coerente com o Plano Nacional de Segurança Viária (PNSv 2001-2016), estabelecido pelo Governo da Colômbia, por meio do Ministério de Transporte, cujo objetivo é reduzir em 50\% a mortalidade derivada dos incidentes de trânsito. Com base nisso, propõe-se uma metodologia para o diagnóstico, planejamento e avaliação das intervenções necessárias para a redução da mortalidade dos atores mais vulneráveis na via.

Palavras-chave: pedestres, segurança viária, modos não motorizados, bicicleta. 


\section{Introducción 1}

De acuerdo con las cifras sobre accidentalidad publicadas por la Secretaria de Movilidad de Medellín [1], en los últimos 5 años han muerto 1500 personas en colisiones de tránsito. De ellos, 760 han sido peatones y 65 han sido ciclistas; del total de las personas fallecidas durante este tiempo los usuarios de modos no motorizados (MNM) representan el 55\%. Coherente con el Plan Nacional de Seguridad Vial (pNsv) 2001-2016 [2],

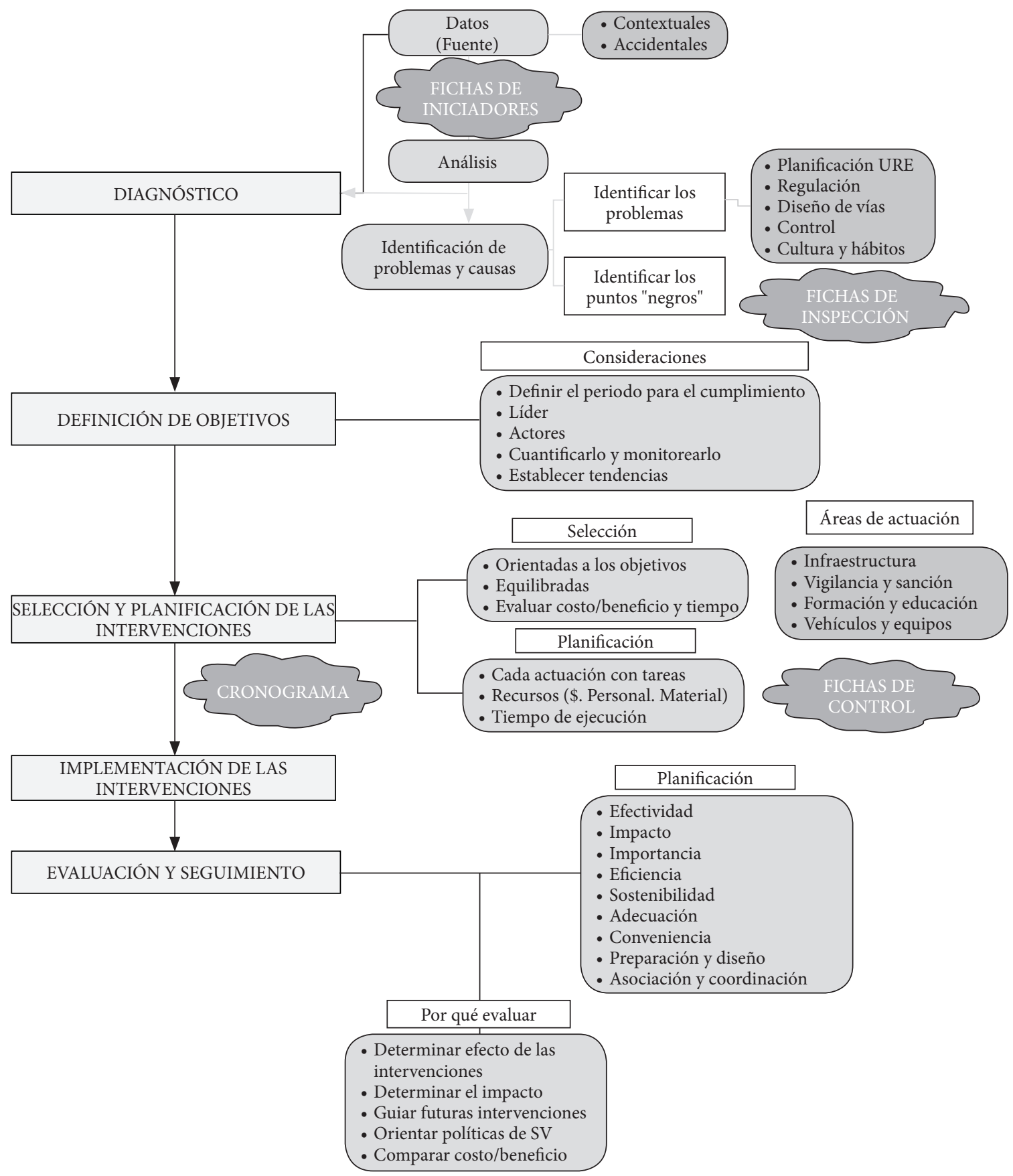

Figura 1. Esquema para la elaboración del PLSV-MNM

Fuente: elaboración propia

1 Este artículo se deriva de una ponencia presentada en el I Congreso Nacional de Vías, realizado en noviembre de 2013, en Medellín (Antioquia), Colombia. 
las ciudades deben diseñar e implementar un plan local de seguridad vial (PLSv) que sea eficaz para la reducción de víctimas y que esté de acuerdo con el contexto de la accidentalidad local. Es dentro de este marco que se propone un plan local de seguridad vial orientado a los actores más vulnerables, es decir, a los peatones y ciclistas. Este plan contiene una metodología para el diagnóstico, la definición de los objetivos, la planificación de las intervenciones, así como la evaluación y seguimiento de las intervenciones seleccionadas.

La participación de los modos no motorizados en la cantidad de muertos para el año 2012 (276 personas) fue de $45 \%$ (123 peatones) y $4 \%$ (11 ciclistas). Comparada con la alta participación de los peatones en las muertes, la proporción para los ciclistas parece baja, pero si se tienen en cuenta la cantidad de desplazamientos que se hacen en bicicleta por día, el resultado indica que este es el modo más peligroso (2,1 muertos por cada millón de desplazamientos en bicicleta, seguido por el motociclista 1,17 y el peatón 0,46 ).

Las colisiones con peatones o ciclistas muertos, entre el 2008 y 2012, han disminuido 21\%, a pesar de que el parque automotor ha aumentado $43 \%$ en el mismo periodo de tiempo. El parque automotor involucrado en una colisión con el peatón para el año 2012 son de tipo: $37 \%$ motos, $37 \%$ autos y $26 \%$ vehículos pesados.
En los siguientes párrafos se presenta la metodología propuesta para el PLSV-MNM, caso Medellín, así como una serie de intervenciones y recomendaciones para la implementación local. En la propuesta se busca el balance entre las medidas para reducir las deficiencias en la infraestructura y las medidas para motivar los cambios de comportamiento seguros. En la figura 1 se indica el esquema para la elaboración del PLSV-MNM.

\section{Diagnóstico [3]}

De las colisiones fatales, los factores de riesgo analizados evidencian que la mayoría de los peatones son personas de la tercera edad, que las colisiones ocurren al final de la tarde y que de los vehículos implicados, motos y automóviles, tienen similar participación (45\%). Estos factores se pueden agrupar en la deficiencia en la infraestructura vial, deficiencia en la planificación urbana y deficiencia en el comportamiento. Con el mapa de la ubicación de las colisiones se puede establecer que para los peatones la "zona negra" es el tramo de la avenida Oriental en el centro de la ciudad, entre las calles 47 y 56 (figura 2); y que para los ciclistas, es el tramo de la Av. Regional entre la calle San Juan y el puente Horacio Toro [4].

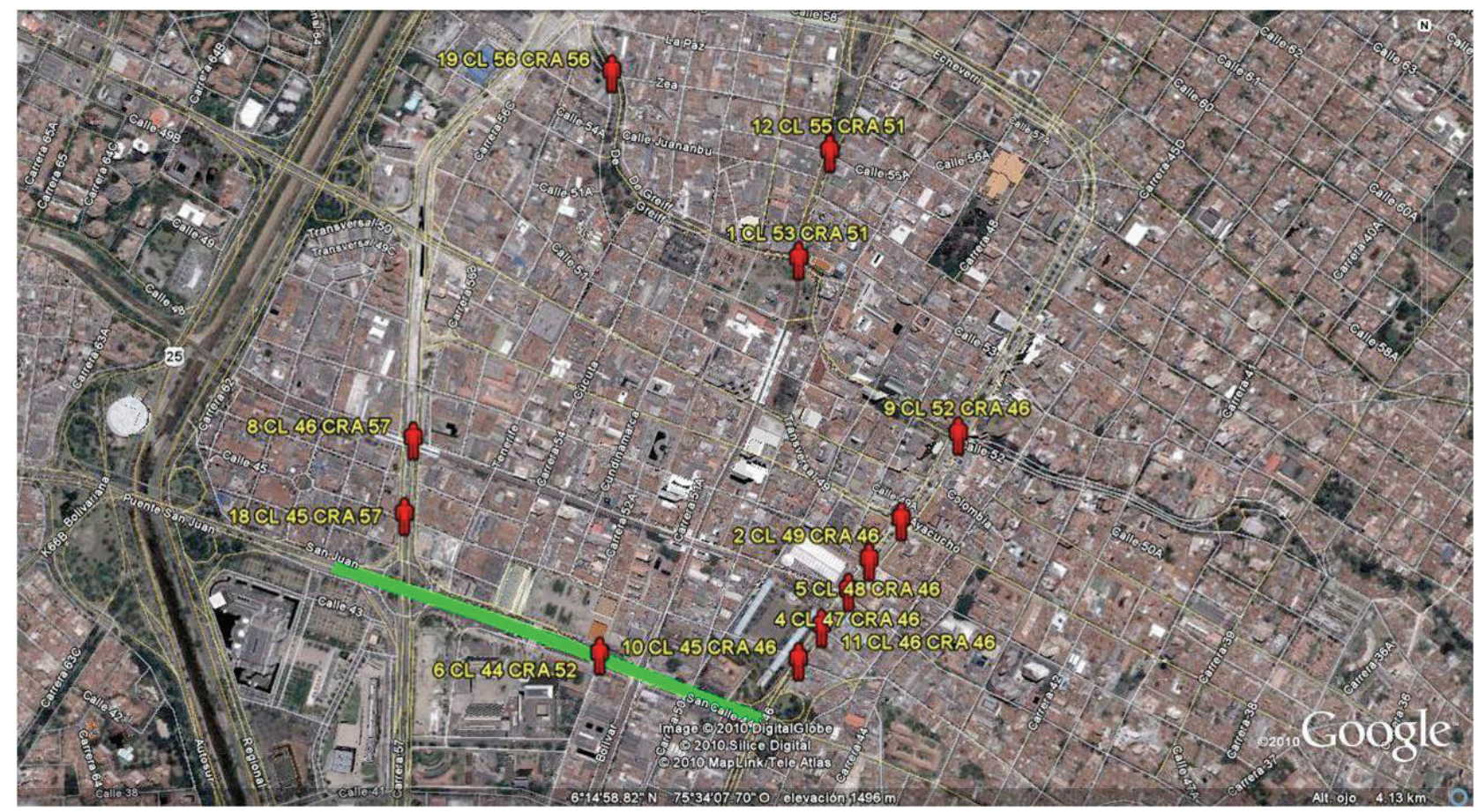

Figura 2. Ubicación de la zona negra de la avenida Oriental (CVPV, 2011)

Fuente: Google Maps y elaboración propia 


\section{Definición de objetivos [5]}

Como objetivo se utiliza el mismo de la Organización Mundial de la Salud (oms, 2011), es decir, una reducción del $50 \%$ en el horizonte 2020. Eso implica que cada año la reducción debe ser en promedio $6 \%$, y pasar de 134 a máximo 67 peatones y ciclistas muertos en colisiones de tránsito que sigue siendo un número alto.

\section{Selección de intervenciones}

El conjunto de intervenciones son principalmente del área de mejoramiento de la infraestructura, control y formación. Estas se presentan de forma numerada:

1. Aumento del tiempo de verde de la fase peatonal y protegiendo el cruce con el aumento del tiempo de seguridad de 3 segundos a 5 segundos. El tiempo mínimo de verde sobre las intersecciones de la avenida Oriental debe tener como mínimo 20 segundos, asegurándose que una persona con una velocidad de 0,9 $\mathrm{m} / \mathrm{s}$ puede cruzarla sin sentir la presión del tiempo que la da la intermitencia al final del verde peatonal.

2. Completar las intersecciones semafóricas que no tienen caras para los peatones.

3. Los planes semafóricos deben cumplir con los tiempos intermedios de despeje.

4. Control con agentes de tránsito para evitar la obstaculización de la cebra por parte de los automotores. Esta situación es muy común en las horas pico, son pocos los que no cruzan aun teniendo el paso en verde si la intersección ya se encuentra bloqueada.

5. Ubicación de los gestores pedagógicos en cada una de las cebras peatonales sobre la avenida Oriental, acompañando el cruce de peatones, especialmente de los ancianos.

6. Reordenamiento de los andenes en el centro de la ciudad, prestando especial atención a la situación de los vendedores informales que están apropiados del espacio público.

7. Operativos de control para los ciclistas que circulan sobre el corredor del río, que es una vía con límite de velocidad de $80 \mathrm{~km} / \mathrm{h}$, múltiples salidas y accesos, con alto tráfico vehicular y participación de vehículos pesados. Estos controles deben orientarse a persuadir al ciclista del alto riesgo que este corre.

8. Gestión de la velocidad sobre el corredor del río, revaluando el límite actual de $80 \mathrm{~km} / \mathrm{h}$ por uno más de acuerdo con las condiciones urbanas que rodean estas vías.

\section{Medidas de acompañamiento}

Las siguientes medidas de acompañamiento tienen como objeto reforzar la implementación de las medidas adoptadas para aumentar la seguridad vial de los MNM y que a la vez sirven para promover los desplazamientos de estos modos como alternativa de movilidad sostenible. Las siguientes medidas tienen una alta posibilidad de ser consideradas debido a las transformaciones actuales del transporte público y en especial gracias a la intención de que Medellín se convierta en la capital latinoamericana de la "Movilidad Sostenible":

- Pacificación del tránsito en zonas de alta afluencia peatonal

- Diseño viario con inclusión para los MNM

- Promoción de las actividades de proximidad

- Densificación alrededor de los corredores del sistema de transporte masivo

- Integración intermodal

- Equipamiento urbano

- Prioridad peatón

- Atención prehospitalaria

- Comportamiento sobre los actores

- Formación en movilidad segura

- Apoyo técnico y financiero

- Voluntad política

\section{Referencias}

[1] Secretaría de Movilidad de Medellín, Informe anual de accidentalidad 2008-2012. Medellín: Colombia, 2012. [En línea]. Disponible en: http://www.medellin.gov.co/ transito/ informes_anuales.html. Último acceso: 2012.

[2] Ministerio de Transporte de Colombia, Plan Nacional de Seguridad social Colombia [En línea]. Disponible en: https://www.mintransporte.gov.co/descargar. php?id=3348. Último acceso: 2013.

[3] Corporación Fondo de Prevención Vial (CFPV), Identificación y propuesta de solución en cinco puntos críticos de accidentalidad de peatones en cinco ciudades colombianas: Informe 1 Análisis de accidentalidad e identificación de puntos críticos. Bogotá, Colombia, 2011.

[4] Secretaría de Movilidad de Medellín, Puntos de mayor accidentalidad, 2012. [En línea]. Disponible: http:// www.medellin.gov.co/transito/puntos_mayor_accidentalidad.html. Último acceso: 2012.

[5] Organización Mundial de la Salud (oms), Pedestrian safety: a road safety manual for decisión makers and practitioners, 2013. [En línea]. Disponible en: http://bit. ly/176LoUM. Último acceso: 2013. 